\title{
Avaliação da regulação sensorial do equilíbrio corporal e risco de queda de mulheres adultas e idosas fisicamente ativas
}

\section{Evaluation of systems of sensory regulation of body balance and risk of fall of adult and elderly physically active \\ Evaluación de la regulación sensorial del equilibrio corporal y riesgo de caída de mujeres adultas y idosas fisicamente activas}

\author{
Marcelo de Maio Nascimento $\left(\mathbb{1}\right.$ ' $\square$, Hugo Daniel Gomez de Castro ${ }^{(1)}$ ', Mateus Alves Ramos $\left({ }^{1}\right.$ \\ Universidade Federal do Vale do São Francisco (UNIVASF). Petrolina, Pernambuco, Brasil.
}

\section{ARTICLE INFO}

\section{Article history}

Received: 05/12/2018

Accepted: 09/04/2019

Published: 29/08/2019

\section{$\triangle$ Correspondent Author} Marcelo de Maio Nascimento

Univ. Federal do Vale do São Francisco Av. José de Sá Maniçoba s/n. - Centro 56304-917, Petrolina, PE, Brasil marcelo.nascimento@univasf.edu.br

\section{(C) 2019 All rights reserved}

Editors

Alfredo Cataldo Neto

Newton Luiz Terra

Assistant Editors

Paula Engroff

Vanessa Sgnaolin

\begin{abstract}
RESUMO
OBJETIVO: Examinar o desempenho sensorial da regulação do equilíbrio estático e dinâmico e o risco de queda de mulheres adultas e idosas, fisicamente ativas.

MÉTODO: Trata-se de um estudo transversal de amostra não probabilística e intencional. Participaram 34 mulheres (51,90 15,84 anos) inscritas em uma academia de ginástica. A população avaliada foi estratificada em faixas etárias: $20-59$ anos $(n=18)$ e 60-69 anos $(n=16)$. A marcha foi avaliada pelos testes Time Up and Go clássico (TUGs), Time Up and Go manual (TUGm) e Time Up and Go cognitivo (TUGc), o equilíbrio pelos testes Dynamic Gait Index (DGI) e Teste de Equilíbrio Corporal (TEC).

RESULTADOS: $O$ teste $t$-Student mostrou diferenças significativas entre grupos etários para as comorbidades: audição, visão, vertigem, labirintite $(p \leq 0,05)$. Comparativamente, mulheres adultas mostraram melhores índices de desempenho que idosas nos testes físicos. Segundo as normativas dos testes, não foi verificado risco de queda para qualquer grupo. Entretanto, independente da faixa etária, o exame detalhado do teste TEC mostrou déficit na regulação sensorial do sistema visual, vestibular e somatossensorial do equilíbrio estático e dinâmico de caidores e não caidores $(p \leq 0,05)$. A análise de regressão logística indicou a regulação interoceptiva do equilíbrio dinâmico como preditor de queda $(p \leq 0,05)$.

CONCLUSÃO: Déficits no sistema vestibular e somatossensorial potencializam paulatinamente o risco de queda de mulheres adultas e idosas, mesmo se mantendo fisicamente ativas.
\end{abstract}

DESCRITORES: Envelhecimento; Equilíbrio Postural; Acidentes por Quedas.

\begin{abstract}
OBJECTIVE: To examine the sensory performance of static and dynamic balance regulation and the risk of falling in physically active adult and elderly women.

METHOD: This is a non-probabilistic and intentional cross-sectional study. Thirty-four women (51.90 \pm 15.84 years) enrolled in a gym attended. The population evaluated was stratified in age groups: $20-59$ years $(n=18)$ and $60-69$ years $(n=16)$. The gait was evaluated by the Time Up and Go (TUGs), Time Up and Go manual (TUGm), and Time Up and Go cognitive (TUGC), the balance by the Dynamic Gait Index (DGI) and Body Balance Test (TEC). RESULTS: Student's t-test showed significant differences between age groups for comorbidities: hearing, vision, vertigo, labyrinthitis $(p \leq 0.05)$. Comparatively, adult women showed better performance indices than older women in physical tests. According to the standards of the tests, there was no risk of falling for any group. However, regardless of age, a detailed examination of the TEC test showed a deficit in the sensory regulation of the visual, vestibular and somatosensory systems of the static and dynamic balance of fallers and non-fallers $(p \leq 0.05)$. The logistic regression analysis indicated the interoceptive regulation of the dynamic balance as a predictor of fall $(p \leq 0.05)$.
\end{abstract}

CONCLUSION: Deficits in the vestibular and somatosensory systems gradually potentiate the risk of falls in adult and elderly women, even if they remain physically active.

KEYwORDS: Aging; Postural Balance; Accidental Falls. 


\section{RESUMEN}

OBJETIVO: Examinar el desempeño sensorial de la regulación del equilibrio estático y dinámico y el riesgo de caída de mujeres adultas y ancianas, físicamente activas.

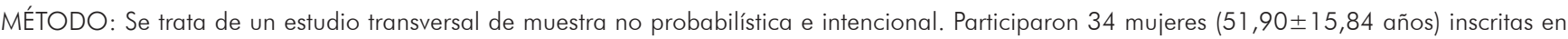
un gimnasio. La población evaluada fue estratificada en grupos de edad: 20-59 años ( $n=18$ ) y 60-69 años ( $n=16$ ). La marcha fue evaluada por las pruebas Time Up and Go clásico (TUGs), Time Up and Go manual (TUGm) y Time Up and Go cognitivo (TUGc), el equilibrio por las pruebas Dynamic Gait Index (DGI) y Test de Equilibrio Corporal (TEC).

RESULTADOS: La prueba t-Student mostró diferencias significativas entre grupos de edad para las comorbilidades: audición, visión, vértigo, laberintitis $(\mathrm{p} \leq 0,05)$. En comparación, las mujeres adultas mostraron mejores índices de rendimiento que los ancianos en las pruebas físicas. Según las normativas de las pruebas, no se verificó riesgo de caída para cualquier grupo. Sin embargo, independientemente del grupo de edad, el examen detallado del test TEC mostró déficit en la regulación sensorial del sistema visual, vestibular y somatosensorial del equilibrio estático y dinámico de cañones y no cañones $(p \leq 0,05)$. El análisis de regresión logística indicó la regulación interoceptiva del equilibrio dinámico como predictor de caída ( $\mathrm{p} \leq 0,05)$.

CONCLUSIONES: Los déficit en el sistema vestibular y somatosensorial potencian paulatinamente el riesgo de caída de mujeres adultas y ancianas, aunque se mantengan físicamente activas.

Palabras ClaVe: Envejecimiento; Balance Postural; Accidentes por Caídas.

\section{INTRODUÇÃO}

O envelhecimento populacional se apresenta como um dos fenômenos deste século. Conforme o Instituto Brasileiro de Geografia e Estatísticaㄹ, no ano de 2030, brasileiros viverão em média 78,6 anos. Estima-se que, em 2040, a expectativa média de vida seja de 79,9 anos. Diante disso, o Brasil será a quinta população mais idosa do planeta, possuindo 19 milhões de habitantes octogenários: indivíduos mais suscetíveis a doenças crônico degenerativas. Entre as questões de saúde pública comuns à população idosa há a queda. Segundo a literatura especializada, $30 \%$ dos idosos do mundo sofreram ou irão sofrer um evento de queda ${ }^{2}$. $\mathrm{O}$ caso é mais evidente em indivíduos acima dos 60 anos e do sexo feminino ${ }^{3}$.

Quedas são responsáveis por lesões, dias de internação, baixando também a percepção da qualidade de vida (QV). Aliado ao fato, quedas elevam os custos do sistema público de saúde 4 . De acordo com Andrade et al. ${ }^{5}$, em 2008, as quedas foram responsáveis no Brasil por 2,18 milhões das hospitalizações de indivíduos acima dos 60 anos. Conforme os autores, no ano de 2030, as quedas serão responsáveis pela hospitalização de 4,91 milhões de idosos. Isso significa dizer, que seus custos de atendimento irão dobrar. Barros e colaboradores ${ }^{6}$ desenvolveram um estudo junto ao banco de dados do Ministério da Saúde brasileiro identificando 399.681 internações de idosos por causa de quedas. Isso foi seguido por custos dos serviços de Autorização e Internação Hospitalar (AIH) de R \$ 464.874.275,91, período de 2005 a 2010.

Assim, com base no rápido envelhecimento populacional e índice de queda independente da faixa etária ${ }^{7}$ é importante o desenvolvimento de estudos que aperfeiçoem as metodologias para o diagnóstico prematuro do risco de queda. Segundo a literatura, quedas sobrevém de fatores extrínsecos ${ }^{8}$ e/ou intrínsecos 9 . Fatores extrínsecos são aqueles relacionados com problemas de iluminação, pavimentação e comportamentos inadequados. Enquanto que causas intrínsecas são aquelas sobrevindas do conjunto de alterações fisiológicas próprias do processo do envelhecimento humano. Por esta razão, quedas são consideradas eventos mutifatoriais, logo sua avaliação exige a aplicação conjunta de diferentes instrumentos.

A literatura especializada destaca a importância do reconhecimento tanto dos indivíduos com alto risco para queda, como os "caidores idiopáticos", aqueles que caem mesmo na ausência de algum pretexto evidente ou enfermidade subjacente ${ }^{10}$. Em se tratando do exame do risco de queda, deve-se considerar que alguns dos instrumentos motores utilizados na área clínica foram validados com populações de sujeitos portadores de comorbidades e/ou dependência funcional. Assim, no caso de indivíduos jovens, adultos, bem como idosos praticantes regulares de exercícios físicos é possível que algumas dessas ferramentas não sejam sensíveis suficientes para detectar o risco de queda, gerando o efeito teto ${ }^{11,12}$. Isso justifica o desenvolvimento de investigações que avaliem e comparem o risco de queda de indivíduos com e sem histórico de queda, igualmente, de diferentes faixas etárias, uma vez que os achados podem ampliar e qualificar os serviços de profissionais da área clínica. Estudos de coorte ${ }^{13}$, revisão sistemática e metanálise ${ }^{14}$ sugeriram a aplicação conjunta de testes de marcha e equilíbrio à estimativa do risco de queda.

Entre os fatores potencializadores do risco há o déficit do equilíbrio corporal. Problemas nesta capacidade motora sobrevém do déficit do desempenho individual e/ou integrado de seus sistemas de regulação 
vestibular, visual e somatossensorial. Este conjunto de sensores são responsáveis pela captação de informações posturais diretamente nas extremidades do corpo, bem como, a emissão dessas informações via aferente ao Sistema Nervoso Central (SNC). Por conseguinte, o SNC processa os dados posturais e emite respostas via eferente às extremidades do corpo, que realizam os ajustes posturais com o auxílio da musculatura ${ }^{15}$. Estudos destacaram, que déficits nesses três sistemas potencializam o risco de queda, principalmente, em idosos ${ }^{10,16}$.

Ainda hoje, a literatura é carente de informações relativas ao déficit do controle postural de indivíduos saudáveis praticantes regulares de exercícios físicos, em especial, dos "caidores idiopáticos"17. Estudos dessa ordem podem auxiliar o entendimento do processo de degeneração do controle postural, além de qualificar as estratégias à prevenção de quedas ${ }^{18}$. $\mathrm{O}$ presente estudo teve como objetivos: i) examinar o desempenho em testes de marcha e equilíbrio de indivíduos adultos e idosos praticantes regulares de exercícios físicos, ii) avaliar o desempenho do sistema sensorial responsável pela regulação do equilíbrio estático e dinâmico, segundo a faixa etária e histórico de queda, e iii) estimar o risco de queda da população avaliada.

\section{METODOLOGIA}

Trata-se de um estudo transversal com amostra não probabilística e intencional. $\mathrm{O}$ cálculo amostral foi realizado pelo Software $G^{*}$ Power 3.1.3, considerando-se $\alpha=0,05$, "Effect size" de $80 \%$ e poder do teste de $80 \%$, o que resultou em 52 participantes: 26 indivíduos para cada grupo. Considerando perda amostral de 30\% foram convidadas 68 indivíduos, estratificados por faixas etárias: G1 (20-59 anos): n=34, e G2 (60-69 ano) $: n=34$. Contudo, a perda amostral foi superior ao esperado, atingindo $50 \%$. Os motivos foram os seguintes: i) determinadas participantes não completaram todas as fases da coleta de dados, ii) por vezes, não apareciam no dia e horário marcado da primeira avaliação, sendo assim, o contato era retomado, algumas também não compareciam a nova data, iii) demais participantes não puderam ser incluídas, em razão dos critérios de inclusão. Diante disso, o tamanho final da amostra foi de 34 indivíduos, divididos em: G1 (20-59 anos): $\mathrm{n}=18$, e $\mathrm{G} 2$ (60-69 anos): $\mathrm{n}=16$.

Todas as participantes estavam inscritas na mesma academia de ginástica, localizada na cidade de Petrolina-PE. Os procedimentos de coleta ocorreram, entre os meses de setembro e outubro de 2018, em ambiente exclusivo, na própria academia. As atividades foram realizadas por dois avaliadores devidamente treinados e familiarizados com os testes. Um avaliador foi responsável por contatar as participantes (mês de agosto, 2018), aplicar os questionários e proceder com o exame antropométrico. Enquanto que o outro realizou exclusivamente os exames da marcha e do equilíbrio. Como critério de inclusão foi adotado não apresentar lesão muscular, articular ou óssea no período da avaliação, possuir tempo mínimo de seis meses de prática de exercícios físicos (musculação ou exercício aeróbico) e visitação mínima de duas vezes por semana na academia. $O$ último critério seguiu as recomendações da Organização Mundial de Saúde $(\mathrm{OMS})^{19}$, que preconiza para idade entre 1864 anos, a realização de atividades de fortalecimento muscular em dois ou mais dias por semana, combinado com 150 minutos de atividade física aeróbica de intensidade vigorosa, ou a associação entre atividades de intensidade moderada e vigorosa. Foram excluídos aqueles que não assinaram o Termo de Consentimento Livre e Esclarecido (TCLE) ou não completaram todas as etapas da investigação. $\mathrm{O}$ estudo foi aprovado pelo Comitê de Ética em Pesquisa com Seres Humanos da Universidade Federal do Vale do São Francisco/ UNIVASF (CAAE: 87416318.8.0000.5196).

Os procedimentos foram realizados em duas fases:

FASE I: Inicialmente, procedeu-se com a coleta das informações sociodemográficas e do autorrelato sobre comorbidades por meio de um questionário construído pela própria equipe, com os seguintes questionamentos:i) idade; ii) comorbidades: problemas de visão, audição, labirintite, vertigem, pressão arterial, Diabetes, osteoporose, reumatismo, esquecimento; iii) histórico de quedas nos últimos 12 meses; e, iv) quantificação dos tipos de medicamento consumidos ao dia. Após isso, procedeu-se com a avaliação antropométrica: A massa corporal foi obtida por meio de uma balança mecânica, até $300 \mathrm{~kg}$ (Welmy, Brasil), enquanto que a estatura foi avaliada com uma régua antropométrica de até 2 metros. Em poder dos dados foi calculado o Índice de Massa Corporal (IMC) por intermédio da fórmula: peso $(\mathrm{kg})$ / estatura $\left(\mathrm{m}^{2}\right)$.

FASE II: Exame do desempenho da marcha/mobilidade e da regulação sensorial do equilíbrio corporal:

\section{Mobilidade}

Três instrumentos foram aplicados: i) Timed Up and Go (TUGs): O TUG clássico avalia a mobilidade e o equilíbrio funcional do indivíduo. Com o indivíduo, inicialmente, sentado em uma cadeira, ele deverá levantar, caminhar e realizar o contorno em um cone colocado a $3 \mathrm{~m}$ de distância retornando até a posição inicial o mais rápido possível, sem correr. O desempenho até 10 segundos é classificado como 
bom, ou seja: sem risco de queda ${ }^{20}$; ii) TUG manual (TUGm): O TUGm consiste na realização da mesma tarefa do TUG clássico. Entretanto, o indivíduo deverá realizar a tarefa transportando uma bandeja de plástico de $25 \mathrm{~cm}$ de diâmetro, com um copo de plástico vazio de $12 \mathrm{~cm}$ de altura. Espera-se indivíduo deverá realizar o percurso sem que o copo caia da bandeja. $O$ ponto de corte utilizado foi de 14,5 segundos $^{21}$; e, iii) TUG cognitivo (TUGc): Nesta terceira variação, o participante realizará o mesmo percurso do TUG clássico, contudo, durante à tarefa foi solicitado a contar de ordem decrescente e em voz alta a partir do número cem. O ponto de corte considerado foi de 15,0 segundos $^{21}$.

\section{Equilíbrio Funcional}

Dynamic Gait Index (DGI)/Índice de Marcha Dinâmica: Com este teste foi avaliado a marcha dinâmica do indivíduo. O DGI possui oito tarefas que simulam atividades da vida diária, como: caminhar na velocidade habitual, sobre uma superfície plana, caminhar alterando a velocidade da marcha, caminhar realizando movimentos horizontais com a cabeça, caminhar realizando movimentos verticais com a cabeça, caminhar e girar sobre o próprio eixo corporal, caminhar e transpor um obstáculo, caminhar e contornar dois cones; subir um lance de escadas. Para a execução do DGI, é necessário realizar marcações no solo com fita adesiva, indicando o ponto de partida, extensão de $1,80 \mathrm{~m}$ e $3,60 \mathrm{~m}$ (onde ficarão os cones) e extensão de $6 \mathrm{~m}$ finalizando o percurso. Cada tarefa do DGI apresenta um escore de zero a três pontos e totalizam o escore de vinte e quatro pontos. No caso de idosos, escores $\leq 19$ pontos estão associados ao risco de queda.

\section{Equilíbrio estático e dinâmico}

O exame da regulação do sistema sensorial, responsável pela regulação do equilíbrio corporal foi executado pelo TEC-Teste de Equilíbrio Corporal, desenvolvido na Alemanha por Wydra ${ }^{22}$ sob o nome de "Gleichgewichtstest" (GGT) e apresentado à comunidade da língua portuguesa por Nascimento et al. ${ }^{23}$. Em seu estudo de validação, o TEC apresentou boa confiabilidade de teste-reteste $(0.78)$, com consistência de alfa Cronbach de 0.92, seguido de correlação de $\mathrm{r}=0.60(<000)$ em radiografias posturais. O TEC é composto por 14 tarefas, dispostas em ordem crescente de dificuldade, com sete tarefas direcionadas para o exame da regulação sensorial do equilíbrio estático e as demais sete para o equilíbrio dinâmico. Nessa perspectiva, o TEC também é capaz de diferenciar o desempenho conforme o tipo da regulação sensorial do equilíbrio, em categorias (Tabela 1): i) EERE: equilíbrio estático regulação exteroceptiva (visual), ii) EERI: equilíbrio estático regulação interoceptiva (vestibular ou somatossensorial), iii) EDRE: equilíbrio dinâmico regulação exteroceptiva (visual), iv) EDRI: equilíbrio dinâmico regulação interoceptiva (vestibular ou somatossensorial). O TEC também apresenta um sistema de normativas à avaliação do desempenho, segundo o sexo e faixas etárias, entre 30-79 anos.

Tabela 1. Disposição das tarefas do TEC, conforme a regulação do equilíbrio.

\begin{tabular}{lll}
\hline Tipo de regulação & Equilíbrio estático & Equilíbrio dinâmico \\
\hline Regulação "Exteroceptiva", & EERE $=1,2,3$ & EERI $=9,10,13$ \\
Regulação "Interoceptiva" & EDRE $=4,6,7,8$ & EDRI $=5,11,12,14$ \\
\hline
\end{tabular}

EERE: equilíbrio estático regulação exteroceptiva; EERI: equilíbrio estático regulação interoceptiva, EDRE: equilíbrio dinâmico regulação exteroceptiva, EDRI: equilíbrio dinâmico regulação interoceptiva.

\section{Descrição das tarefas do TEC}

\section{EERE (Equilíbrio Estático Regulação Exteroceptiva)}

Tarefa 1: $\mathrm{O}$ indivíduo, apoiado somente sobre um dos membros inferiores (livre escolha) deve permanecer nesta posição, sem movimentar o pé de apoio por pelo menos 15 segundos.

Tarefa 2: $\mathrm{O}$ indivíduo, apoiado somente sobre um dos membros inferiores (livre escolha) deve oscilar a outra perna para frente e para trás, sem movimentar o pé de apoio, permanecendo nesta posição por pelo menos 15 segundos.

Tarefa 3: $\mathrm{O}$ indivíduo deverá realizar um giro de $360^{\circ}$ e depois ficar apoiado somente sobre um dos membros inferiores (livre escolha) sem movimentar o pé de apoio, por pelo menos 15 segundos.

\section{EERI (Equilíbrio Estático Regulação Interoceptiva)}

Tarefa 9: $\mathrm{O}$ indivíduo deve caminhar sobre a trave (4 metros de comprimento, $10 \mathrm{~cm}$ de largura e $3 \mathrm{~cm}$ de altura, incluindo) mantendo o equilíbrio até o final da trave.

Tarefa 10: $\mathrm{O}$ indivíduo deve caminhar por 2 metros sobre a trave, girar $180^{\circ}$ e retornar ao início da trave, mantendo o equilíbrio até o final do percurso.

Tarefa 13: $\mathrm{O}$ indivíduo deve caminhar sobre a trave e simultaneamente quicar uma bola de voleibol sobre o solo, ao seu lado, mantendo o equilíbrio até parar no final da trave. 


\section{EDRE (Equilíbrio Dinâmico Regulação Exteroceptiva)}

Tarefa 4: $\mathrm{O}$ indivíduo, apoiado somente sobre um dos membros inferiores (livre escolha) deve movimentar o outro pé no ar em volta de duas maças de ginástica rítmica ou garrafas de plástico desenhando o número 8. Os objetos devem estar posicionados lateralmente ao seu corpo, distantes um do outro na mesma medida de suas alturas. A tarefa deverá ser realizada sem que o pé toque o chão ou os objetos, primeiro com os olhos abertos, em seguida fechados.

Tarefa 6: O indivíduo, com os olhos fechados, apoiado somente em um dos membros inferiores (livre escolha), deve permanecer nessa posição, sem movimentar o pé de apoio, por pelo menos 15 segundos.

Tarefa 7: O indivíduo deverá realizar um giro de $360^{\circ}$, fechar os olhos e a seguir ficar apoiado somente sobre um dos membros inferiores (livre escolha) sem movimentar o pé de apoio, por pelo menos 15 segundos.

Tarefa 8: O indivíduo, com os olhos fechados, apoiado somente em um dos membros inferiores (livre escolha) deve oscilar a outra perna para frente e para trás, sem movimentar o pé de apoio, permanecendo nesta posição por pelo menos 15 segundos.

\section{EDRI (Equilíbrio Dinâmico Regulação Interoceptiva)}

Tarefa 5: O indivíduo, com os olhos fechados, deverá realizar cinco vezes o movimento de polichinelo sobre uma linha, movimentando os braços e pernas simultaneamente. Ao final da execução de cada movimento pelo menos um dos pés deverá estar em contato com a linha.

Tarefa 11: $\mathrm{O}$ indivíduo deve caminhar por $2 \mathrm{~m}$ de costas sobre a trave, girar $180^{\circ}$ e terminar o percurso de frente mantendo o equilíbrio até parar no final da trave.

Tarefa 12: $\mathrm{O}$ indivíduo deve caminhar por $2 \mathrm{~m}$ de costas sobre a trave, realizar um giro completo de $360^{\circ}$ e terminar o percurso retornando a posição de origem, mantendo o equilíbrio até parar no final da trave.

Tarefa 14: O indivíduo deve caminhar com os olhos fechados sobre a trave, mantendo o equilíbrio até parar no final da trave.

\section{Procedimentos estatísticos}

A normalidade dos dados foi obtida pelo teste de Shapiro Wilk. O teste do Qui-quadrado foi utilizado à comparação das variáveis nominais, na presença de valores menores que cinco, aplicou-se o teste exato de Fisher. O teste $t$-Student para amostras independentes foi utilizado para determinar os níveis de significância das variáveis investigadas nas comparações entre faixas etárias (20-59 anos e 60-69 anos) e grupos
(Não-caidor e Caidor). Com a análise de regressão linear multivariada das dimensões do teste TEC (EERE, EERI, EDRE, EDRI) foram identificadas como preditoras de queda, considerou-se para o cálculo apenas aquelas que apontaram níveis de significância no modelo univariado. A ordem de inserção foi do maior para o menor (modelo forward), respeitando a magnitude do coeficiente de correlação de Spearman. As razões de chances (OR, IC 95\%) foram utilizadas à apresentação dos resultados. Os dados foram processados no programa estatístico SPSS, versão 22.0. O nível de significância adotado foi de $\mathrm{p} \leq 0,05$.

\section{RESULTADOS}

Tabela 2. Principais características da população avaliada

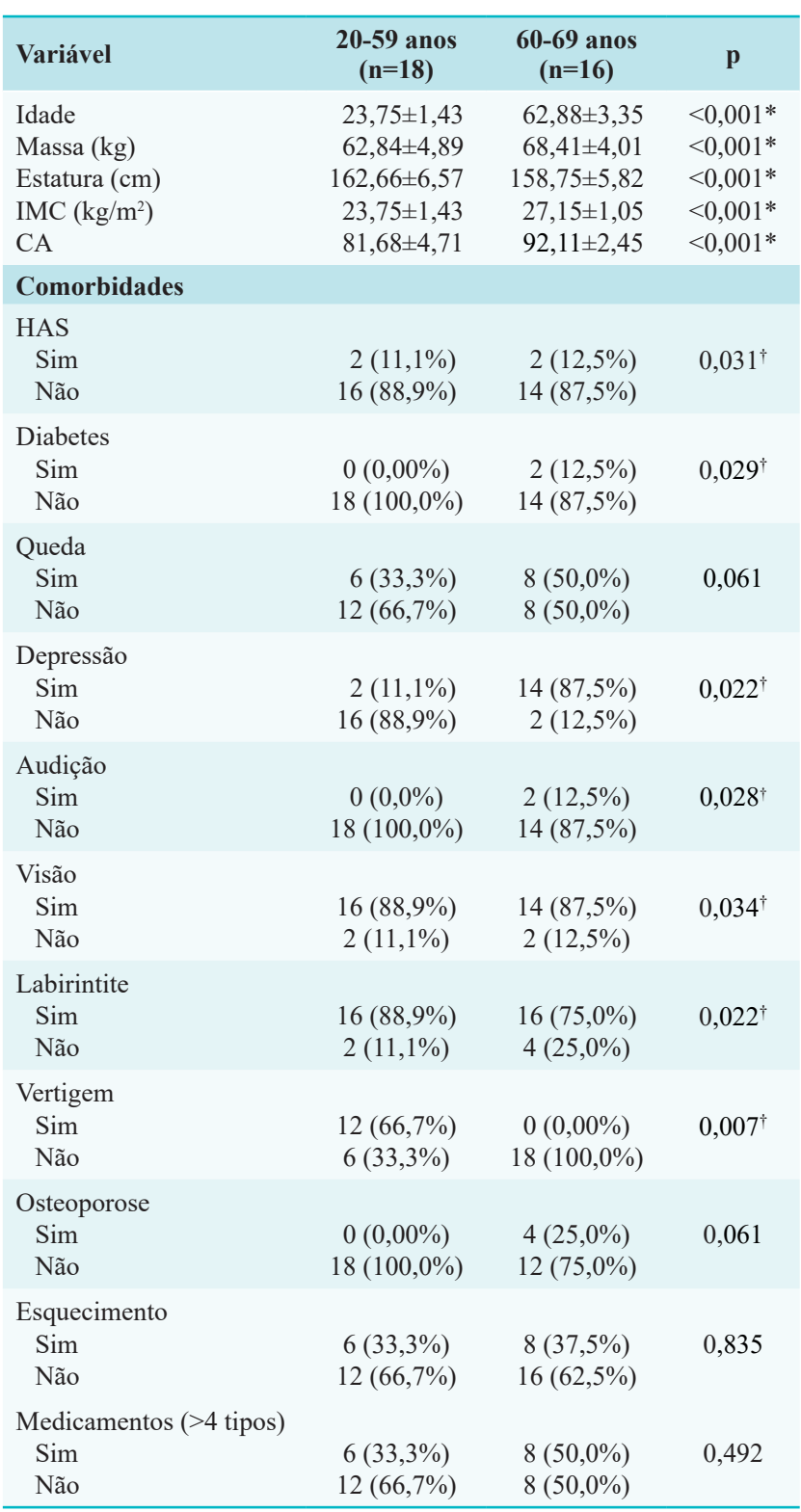

IMC: Índice de Massa Corporal, kg: quilograma, cm: centímetros, $\mathrm{kg} / \mathrm{m}^{2}$ : Quilograma por metro quadrado, CA: circunferência abdominal, HAS: hipertensão arterial, *p $\leq 0,05$ Mann Whitney, ${ }^{\dagger} \mathrm{p} \leq 0,05$ Qui-quadrado. 
A Tabela 2 apresenta as principais características da população avaliada, segundo as faixas etárias. Resultados significativos foram observados para ambos os grupos nas variáveis idade, massa corporal, estatura, IMC e circunferência abdominal $(p \leq 0,05)$. Verificouse maior índice do IMC e circunferência abdominal entre as participantes em idade $\geq 60$ anos. Este grupo também mostrou resultados diferentemente estatísticos para as comorbidades Diabetes, hipertensão arterial e depressão $(\mathrm{p} \leq 0,05)$. Ademais, observou-se níveis de significância junto ao conjunto de variáveis associadas ao risco de queda: audição, visão, vertigem e labirintite também mostraram níveis de significância $(\mathrm{p} \leq 0,05)$.

Tabela 3. Resultados das dimensões do teste TEC, segundo faixas etárias

\begin{tabular}{lccc}
\hline Variáveis & $\begin{array}{c}\mathbf{2 0 - 5 9} \text { anos } \\
(\mathbf{n = 1 8})\end{array}$ & $\begin{array}{c}\mathbf{6 0 - 6 9} \text { anos } \\
(\mathbf{n = 1 6})\end{array}$ & $\mathbf{p}$ \\
\hline TUGs & $8,63 \pm 0,86$ & $9,73 \pm 1,30$ & 0,588 \\
TUGm & $9,85 \pm 1,16$ & $11,07 \pm 1,94$ & 0,327 \\
TUGc & $10,12 \pm 1,73$ & $11,37 \pm 4,08$ & 0,442 \\
DGI & $23,44 \pm 1,01$ & $23,38 \pm 1,40$ & 0,724 \\
TEC-Total & $10,11 \pm 1,27$ & $7,88 \pm 3,18$ & 0,160 \\
Dimensões do TEC & & & \\
$\quad$ EERE (3 tarefas) & $2,88 \pm 0,33$ & $2,12 \pm 1,12$ & $0,009^{*}$ \\
EERI (4 tarefas) & $1,77 \pm 1,39$ & $1,12 \pm 0,83$ & $0,022^{*}$ \\
EDRE (3 tarefas) & $2,77 \pm 0,44$ & $2,25 \pm 1,03$ & 0,105 \\
EDRI (4 tarefas) & $2,66 \pm 0,70$ & $2,37 \pm 0,91$ & $0,004^{*}$ \\
\hline
\end{tabular}

EERE: equilíbrio estático regulação exteroceptiva, EERI: equilíbrio estático regulação interoceptiva, EDRE: equilíbrio dinâmico regulação exteroceptiva, EDRI: equilíbrio dinâmico regulação interoceptiva.

* $\mathrm{p} \leq 0,05$ Mann Whitney.
A Tabela 3 apresenta os resultados médios do desempenho, segundo faixas etárias, para o exame da marcha e equilíbrio. Comparativamente, mulheres mais jovens mostraram melhores escores nos testes motores. Entretanto, considerando o nível de significância $(p \geq 0,05)$ não foi possível afirmar a superioridade do desempenho de mulheres de 20-59 anos sobre aquelas da faixa dos 60-69 anos. Em se tratando da avaliação da regulação sensorial do equilíbrio estático e dinâmico, as dimensões EERE, EERI, EDRI mostraram níveis de significância $(\mathrm{p} \leq 0,05)$.

A Figura 1 mostra comparativamente os escores do desempenho das participantes nas quatro dimensões do teste TEC. De acordo com os resultados, o grupo não caidor indicou melhor escore de desempenho nas quatro dimensões do TEC (Fig. 1A). Comparativamente, o maior déficit do desempenho foi constatado na dimensão responsável pelo ajuste interoceptivo do equilíbrio estático $(p \leq 0,05)$. O confronto do desempenho das dimensões do TEC, conforme faixas etárias, é mostrado na Fig. 1B. Comparativamente, o grupo mais jovem mostrou melhor desempenho, corroborando com a análise entre caidores e não caidores (Fig. 1A), a dimensão EERI exibiu o maior déficit para ambos os grupo $(p \leq 0,05)$. Ademais, resultados diferentemente significativos também foram observados para o conjunto de tarefas EERE e EDRI $(\mathrm{p} \leq 0,05)$.

Com a análise de regressão linear múltipla foram estimadas as tarefas do teste TEC previsoras de quedas (Tabela 4). A inserção das variáveis no modelo

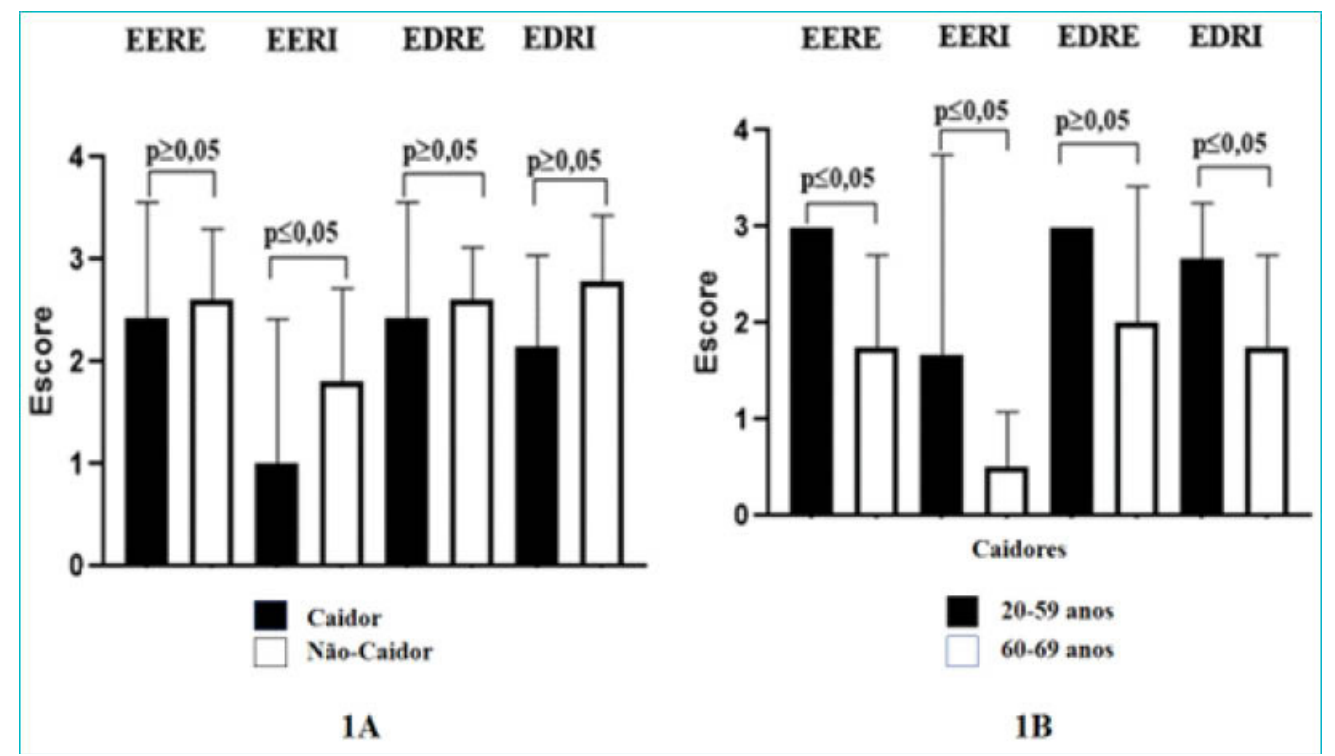

Figura 1. Distribuição média do escore do desempenho da regulação sensorial do equilíbrio estático e dinâmico. EERE: equilíbrio estático regulação exteroceptiva, EERI: equilíbrio estático regulação interoceptiva, EDRE: equilíbrio dinâmico regulação exteroceptiva, EDRI: equilíbrio dinâmico regulação interoceptiva, $\mathrm{p} \leq 0,05$ Mann Whitney. 
ocorreu de forma agrupada, conforme as dimensões do teste: EERE, EERI, EDRE, EDRI. O modelo obtido foi estatisticamente significativo $[\mathrm{F}(4,12)=0,354$; $\left.p=0,042 ; R^{2}=0,357\right]$. Já o modelo final de probabilidade de queda apresentado revelou unicamente a dimensão EDRI $(\beta=-0,552 ; \mathrm{t}=-2,096 ; \mathrm{p} \leq 0,05)$ como previsora do risco de queda da população avaliada. Tarefas agrupadas nesta dimensão são responsáveis pelo ajuste interoceptivo do equilíbrio dinâmico.

Tabela 4. Resultados do modelo de regressão das dimensões sensoriais do teste TEC

\begin{tabular}{lccc}
\hline Variável & B & Odds ratio $(\mathbf{9 5 \%})$ & $\mathbf{p}$ \\
EDRI & $-0,350$ & $-0,552(0,714-0,014)$ & $0,049^{*}$ \\
EDRE & 0,172 & $0,272(-0,290-1,634)$ & 0,432 \\
EERE & 0,023 & $0,040(-0,365-1,012)$ & 0,899 \\
EERI & $-0,200$ & $-0,466(-0,441-1,040)$ & 0,094 \\
\hline
\end{tabular}

EERE: equilíbrio estático regulação exteroceptiva, EERI: equilíbrio estático regulação interoceptiva, EDRE: equilíbrio dinâmico regulação exteroceptiva, EDRI: equilíbrio dinâmico regulação interoceptiva.

$* \mathrm{p} \leq 0,05$.

\section{DISCUSSÃO}

O presente estudo avaliou o desempenho de mulheres adultas e idosas em testes de marcha e equilíbrio. Conforme os escores do desempenho dos três testes de marcha (TUGs, TUGm, TUGc) não foi constatado risco de queda para as integrantes de ambos os grupos. A interpretação dos escores dos testes de equilíbrio corporal (DGI e TEC) também não apontou risco de queda. Por outro lado, a análise do desempenho dos grupos nas dimensões do teste TEC ofereceu um exame mais detalhado sobre a condição sensorial do controle postural. Na comparação do desempenho, verificou-se diferenças estatisticamente significativas para dimensão EERI, segundo o histórico de quedas. O mesmo foi observado nas dimensões EERE, EERI e EDRI para o confronto entre caidores por faixas etárias. Os achados são importantes, visto que tanto o exame realizado pelos três testes de marcha TUG, como a pontuação total dos testes de equilíbrio DGI e TEC não apontaram risco para queda.

No presente estudo, diferenças estatisticamente significativas foram observadas para a idade e índices antropométricos. Conforme a análise dos resultados do IMC e da circunferência abdominal, participantes de ambos os grupos se mostraram dentro dos parâmetros de saúde da OMS. ${ }^{24}$ Entre as comorbidades, o grupo em idade $\geq 60$ anos indicou prevalência de 87,5\% para depressão, seguido de níveis de significância. $\bigcirc$ caso merece atenção, pois a depressão está associada à perda de peso e humor, sentimento de culpa, ideação suicida, queixa de dores, eventualmente, psicose e hipocondria. ${ }^{25}$ Em relação ao consumo de medicamentos, não foi possível constatar consumo superior a quatro tipos medicamentos ao dia (polifarmácia) nas participantes do grupo de 20-59 anos. O grupo entre 60-69 anos apresentou consumo de medicação considerada normal para sua faixa etária. Segundo a OMS, ${ }^{26}$ a depressão é um transtorno mental, cada vez mais frequente na população mundial, estima-se que no ano de 2030, ela seja a principal causa da incapacidade mental. Por esta razão, ao final do estudo, as participantes além de receberem relatórios sobre seus desempenhos motores e risco de queda, também foram informadas sobre seu estado de saúde mental.

Com base nos resultados, comparativamente, participantes jovens (20-59 anos) indicaram os melhores escores nos testes de mobilidade e equilíbrio. $\mathrm{O}$ fato se apresenta como uma consequência natural da fisiologia humana, que faz com que células mais jovens sejam capazes de metabolizar energia mais rapidamente. A associação disso com um aparelho muscular e sensorial preservado oferece maior vigor à realização de tarefas físicas. ${ }^{27} \mathrm{~A}$ interpretação do desempenho da população avaliada nos testes de mobilidade e equilíbrio não indicou risco de queda. Uma possível explicação para o fato pode estar associada à prática do exercício físico, que se apresenta como fator protetor de quedas. . $^{28,29}$

Em idade avançada, tanto o diâmetro das fibras musculares, como os níveis de força muscular diminuem, gerando fraqueza, diminuindo a velocidade de reação, o que, por conseguinte, potencializa o risco de quedas. $O$ treinamento de musculação $2-3$ vezes por semana é capaz, por exemplo, de aumentar ou manter os níveis de força tanto de indivíduos jovens, como de idosos..$^{30} \mathrm{O}$ níveis satisfatórios de desempenho motor das participantes do estudo nas avaliações pode, de tal modo, decorrer dos efeitos do treinamento de força capaz tanto de amentar a velocidade da marcha ${ }^{31}$, como de beneficiar o controle postural. ${ }^{32}$ Por outro lado, sabe-se que apenas o treinamento de musculação não é suficiente para reduzir as chances de queda, uma vez que a questão é multifatorial.

Em relação ao autorrelato de quedas, a maior frequência foi observada entre o grupo das idosas. O fato corrobora com a literatura especializada ${ }^{33}$. Por outro lado, observou-se também que $1 / 3$ do grupo de mulheres adultas mostraram histórico de queda. Em estudo desenvolvido por Alves et al. ${ }^{3}$ com idosos da comunidade, 206 indivíduos ( $\geq 60$ anos) foram entrevistadas sobre fatores associados à queda, desses $36,4 \%$ atestaram histórico de quedas. Comparativamente, no presente estudo, a taxa de 
queda entre as sexagenárias foi superior $(50,0 \%)$. Com respeito ao déficit do controle postural, Teixeira ${ }^{34}$ afirma que independente da faixa etária, sua eficácia está relacionada ao funcionamento do sistema sensorial, representado pelo aparelho vestibular, visual e somatossensorial. Nessa perspectiva, deve-se considerar, igualmente, como fator potencializador do risco de cair o conjunto de comorbidades apresentadas pelo indivíduo ${ }^{35}$. No presente estudo, verificou-se diferenças estatisticamente significativas para as comorbidades visão, audição, vertigem e labirintite, todas relacionadas com aumento do risco de queda.

Em estudo de revisão sistemática e metanálise sobre a determinação do risco de queda de adultos idosos, que incluiu 2294 estudos, publicados entre 1990-2013 ${ }^{14}$, após análise de 59 investigações, os autores destacaram a suscetibilidade da interação de múltiplos fatores na questão da queda. Dentre eles foi apontado a condição funcional dos sistemas sensoriais visão, audição e propriocepção, intitulados como fatores intrínsecos. Nessa perspectiva, Rodrigues et al..$^{36}$ salientaram, que devido ao processo do envelhecimento, o sistema visual tende a apresentar diminuição da acuidade e do campo visual, limitando a capacidade de adaptação do indivíduo ao escuro, aumentando o limiar de percepção à luminosidade. Outro ponto em questão é a degeneração das fibras proprioceptivas, que se encontram associadas à sensibilidade cinestésica. Do ponto auditivo, as fibras sinápticas sofrem alterações eletrofisiológicas, o que é sobrevindo do avanço do atrito entre as fibras nervosas e o nervo vestibular. Todas essas alterações, independentemente da idade e histórico de quedas do indivíduo, podem potencializar consideravelmente o risco de quedas futuras ${ }^{10,37}$.

Em outro estudo de revisão sistemática, que selecionou 87 estudos, entre 1995-2010, com foco nas consequências físicas de quedas de idosos, os autores evidenciaram sobre o aumento das disfunções na integração de informações aferentes e eferentes junto ao Sistema Nervoso Central (SNC) ${ }^{38}$. Isso pode se apresentar como um impedimento para que o SNC envie informações posturais corretivas, em tempo hábil às extremidades. No presente estudo, verificouse $88,9 \%$ de prevalência do déficit visual junto ao grupo de adultos (20-59 anos) e 87,5\% entre os idosos. O aparelho visual, apresenta-se, por exemplo, como a primeira fonte reguladora da intensidade das oscilações corporais $^{39}$ Mediante informações visuais periféricas e centrais, o SNC orienta o posicionamento do corpo no espaço, a partir de um sistema de relação entre os eixos horizontais e verticais do corpo e objetos dispostos no entorno. Diante disso, a frequência das oscilações sobre a base de apoio é minimizada ${ }^{40}$. Na ausência da informação visual as oscilações corporais aumentam, assim como o desequilíbrio ${ }^{37}$.

Verificou-se, também, déficit das participantes no conjunto de tarefas responsáveis pela regulação interoceptiva do equilíbrio estático (EERI) e dinâmico (EDRI). Os itens dessas tarefas possuem íntima relação com o aparelho vestibular e somatossensorial. $\mathrm{O}$ fato merece atenção, visto que indivíduos com transtornos vestibulares podem apresentar conflito sensorial e retardo nos ajustes posturais. Segundo Quitschal e Fukunaga ${ }^{41}$, a vertigem é um dos principais problemas vestibulares da população adulta e idosa, responsável por cerca de $50 \%$ dos casos de distúrbio do equilíbrio.

A análise de regressão logística indicou como previsor de queda, o déficit do desempenho do sistema de regulação sensorial interoceptivo do equilíbrio dinâmico (EDRI). Com base no resultado da razão de chance (OR), pode-se dizer que o bom desempenho do sistema vestibular e somatossensorial causa efeito protetor sobre quedas, capaz de reduzir em $45 \%$ a chance de que o indivíduo caia. $\mathrm{O}$ achado corrobora com o estudo de Lemos, David e Mota ${ }^{42}$, que compararam o controle postural de mulheres adultas 19-30 anos identificando baixa do controle postural, principalmente, em situações de obstrução da visão. Toledo e Barela ${ }^{43}$ analisaram o equilíbrio de jovens e idosos, constatando déficits do sistema proprioceptivo de ambos os grupos. As quatro tarefas da dimensão EDRI assinaladas como previsoras de queda demandam o controle postural durante à movimentação sobre uma superfície reduzida, com troca de direção, seguido pela marcha para trás. Nessa perspectiva, constatou-se que tanto mulheres adultas, como idosas apresentaram déficits comuns em canais sensoriais vestibulares e proprioceptivos. $\mathrm{O}$ achado além de importante apresenta implicações clínicas terapêuticas para o planejamento dos exercícios da população avaliada, além de sugestões para o cuidado em saúde de demais indivíduos da comunidade. Sendo assim, seria conveniente a conscientização dos envolvidos sobre os déficits funcionais graduais sofridos pelo sistema sensorial, em função do avanço da idade. Isso implica na realização de exames periódicos das funções sensoriais envolvidas no ajuste postural.

Algumas limitações do presente estudo devem ser consideradas. Primeiro, o pequeno número de participantes e seu desenho transversal, que dificultam a generalização dos resultados. Em segundo lugar, não foi possível controlar a dose do treinamento de força e aeróbico realizado pelas participantes, pelas seguintes razões: i) todas apresentaram diferença no tempo da prática dos exercícios (6-28 meses), ii) possuíam fichas com rotinas de treinamento pró- 
prio, iii) não apresentaram homogeneidade quanto os dias de visitação a academia (2-5 dias por semana) e, iv) diferente tempo de treinamento diário foram observados, variação de 30-90 minutos por seção. Outra limitação consistiu no questionário de comorbidades, que não detalhou de modo exato a tipologia dos problemas visuais e vestibulares relatados.

\section{CONCLUSÃO}

No estudo em questão, como esperado mulheres do grupo mais jovem (20-59 anos) apresentaram melhor desempenho no exame da marcha e do equilíbrio. Independente da faixa etária, verificou-se para caidoras e não caidoras prevalência de comorbidades capazes de potencializar o risco de queda, visto que comprometem a integração de estímulos sensoriais do aparelho vestibular, somatossensorial e visual. Segundo a interpretação dos resultados finais dos testes clínicos, não foi detectado risco de queda para qualquer faixa etária. Em contra partida, o exame detalhado da condição sensorial da regulação do equilíbrio estático e dinâmico, oferecido pelas dimensões do teste TEC identificou, que independente da faixa etária e prática regular de exercícios físicos, a população avaliada apresentou alterações no funcionamento da regulação sensorial exteroceptiva (EERE e EERI) e interoceptiva (EDRI). Diante dos achados, sugere-se aos profissionais da área da saúde, que incorporem nos exames periódicos a avaliação das funções sensoriais responsáveis pela regulação do equilíbrio corporal. Aconselha-se também o desenvolvimento de estudos futuros, que valorizem o acompanhamento longitudinal, uma vez que a medida possibilitaria constatar se os resultados exibidos pela regressão logística são realmente um fator preditor de quedas futuras, bem como, identificaria o momento em que os déficits sensoriais se tornam sintomáticos.

\section{REFERÊNCIAS}

1. Instituro Brasileiro de Geografia e Estatística. Projeção da população do Brasil por sexo e idade: 2000-2060 [Internet]. Rio de Janeiro: IBGE; 2018 [capturado em 27 maio 2019]. Disponível em: https://ww2.ibge.gov.br/ home/estatistica/populacao/projecao_da_populacao/2013/ default.shtm. https://doi.org/10.1160б/d.17.2019.tde17102018-135239

2. Tinetti M, Kumar C. The patient who falls: "It's always a trade-off." Jama. 2010;303(3):258-66. https://doi. org/10.1001/jama.2009.2024

3. Alves RLT, Silva CFM, Pimentel LN, Costa IA, Souza ACS, Coelho LAF. Avaliação dos fatores de risco que contribuem para queda em idosos. Rev Bras Geriatr Gerontol. 2017;20(1):59-69. https://doi.org/10.1590/s1809. 98232011000400015
4. Moraes AS, Soares WJS, Lustosa LP, Bilton TL, Ferrioli E, Perracini MR. Características das quedas em idosos que vivem na comunidade: estudo de base populacional. Rev Bras Geriatr Gerontol. 2017;20(5):693-704. https://doi.org/10.1590/s1809-98232014000100006

5. Andrade IR, Souza EA, Luz LA, Pinto Júnior EP. Características e gastos com hospitalizações por quedas em idosos. J Heal Sci Inst. 2017;35(1):28-31.

6. Barros IFO, Pereira MB, Weiller TH, Anversa ETR. Internações hospitalares por quedas em idosos brasileiros e os custos correspondentes no âmbito do Sistema Único de Saúde. Rev Kairós Gerontol. 2015;18(4):63-80. https://doi.org/10.21902/jhmreview.v2i1.321

7. Santos RKM, Maciel ACC, Britto HMJS, Lima JCC, Souza TO. Prevalência e fatores associados ao risco de quedas em idosos adscritos a uma Unidade Básica de Saúde do município de Natal, RN, Brasil. Cien Saude Colet. 2015;20(12):3753-62. https://doi.org/10.1590/1413 812320152012.00662015

8. Gonçalves AK, Hauser E, Martins VF, Possamai VD, Griebler EM, Blessmann EJ, Teixeira AR. Postural balance program: variables related to falls in elderly. J Phys Educ. 2017;28:e2808. https://doi.org/10.4025/jphyseduc.v28i1.2808

9. Gil AWO, Silva RA, Oliveira MR, Carvalho CE, Oliveira DAAP. Comparação do controle postural em cinco tarefas de equílibrio e a relação dos riscos de quedas entre idosas e adultas jovens. Fisioter Pesqui. 2017;24(2):120-6. https://doi.org/10.1590/1809-2950/15804424022017

10. Fasano A, Plotnik M, Bove F, Berardelli A. The neurobiology of falls. Neurol Sci. 2012;33(6):1215-23. https://doi.org/10.1007/s10072-012-1126-6

11. Karuka AH, Silva JAMG, Navega MT. Análise da concordância entre instrumentos de avaliação do equilíbrio corporal em idosos. Rev Bras Fisioter. 2011;15(6):460-6. https://doi.org/10.1590/s1413. 35552011000600006

12. Santos GM, Souza ACS, Virtuoso JF, Tavares GMS, Mazo GZ. Valores preditivos para o risco de queda em idosos praticantes e não praticantes de atividade física por meio do uso da Escala de Equilíbrio de Berg. Rev Bras Fisioter. 2011;15(2):95-101. https://doi.org/10.1590/s1809. 29502009000100002

13. Eagles D, Perry JJ, Sirois MJ, Lang E, Daoust R, Lee J, Griffith L, Wilding L, Neveu X, Emond M. Timed up and go predicts functional decline in older patients presenting to the emergency department following minor trauma. Age Ageing. 2017;46(2):214-8. https://doi.org/10.1093/ ageing/afw184

14. Lusardi MM, Fritz S, Middleton A, Allison L, Wingood M, Phillips E, Criss M, Verma S, Osborne J, Chui KK. Determining risk of falls in community dwelling older adults: a systematic review and meta-analysis using posttest probability. J Geriatr Phys Ther. 2017;40(1): 1-36. https://doi.org/10.1519/jpt.0000000000000099

15. Lanzarin M, Parizzoto P, Libardoni TC, Sinhorim L, Tavares GMS, Santos GM. A influência da dupla tarefa no controle postural de adultos jovens. Fidioter Pesqui. 2015;22(1):61-8. 
16. Leme GLM, Carvalho IF, Scheicher ME. Melhora do equilíbrio postural em mulheres idosas com o uso de informação sensorial adicional. Fisioter Pesqui. 2017;24(1):68-73. https://doi.org/10.1590/1809. 2950/16753224012017

17. Lesinski M, Hortobágyi T, Muehlbauer T, Gollhofer A, Granacher U.. Effects of balance training on balance performance in healthy older adults: a systematic review and meta-analysis. Sport Med. 2015;45(12):1721-38. https://doi.org/10.1007/s40279-015-0375-y

18. Shier V, Trieu E, Ganz DA. Implementing exercise programs to prevent falls: systematic descriptive review. Inj Epidemiol. 2016;3(1):16. https://doi.org/10.1186/ s40621-016-0081-8

19. World Health Organization. Global Recommendations on physical activity for Health [Internet]. Geneva: WHO; 2010 [capturado em 27 mio 2019]. Disponível em: https://www.who.int/dietphysicalactivity/factsheet_ recommendations/en/.

20. Podsiadlo D, Richardson S. The time "Up \& Go": a test of basic functional mobility for frail elderly persons. J Am Geriatr Soc. 1991;39(2):142-8. https://doi. org/10.1111/j.1532-5415.1991.tb01616.x

21. Shumway-Cook A, Brauer S, Woollacott M. Predicting the Probability for falls in community-dwelling older adults using the timed up \& go test. Phys Ther. 2000;80(9): 896-903. https://doi.org/10.1093/ptj/80.9.896

22. Wydra G. Bedeutung, diagnose und therapie von gleichgewichtstörung. Motorik. 1993;16(3):100-7.

23. Nascimento M, Appell IC, Coriolano H-JA. Teste de equilíbrio corporal (TEC) para idosos independentes. RPCD. 2012;12(2):71-82. https://doi.org/10.5628/ rpcd.12.02.71

24. World Health Organization. Physical status: the use and interpretation of anthropometry. Geneva: WHO, 1998.

25. Moraes H, Deslandes A, Ferreira C, Pompeu FAMS, Ribeiro P, Laks J. O exercício físico no tratamento da depressão em idosos: revisão sistemática. Rev Psiquiatr RS. 2007;29(1):70-9. https://doi.org/10.1590/s010181082007000100014

26. World Health Organization. Depression: a global crisis [Internet]. Geneva: WHO; 2012 [capturado em 27 maio 2019]. Disponível em: http://www.who.int/mental_health/ management/depression/wfmh_paper_depression wmhd_2012.pdf.

27. World Health Organization. Ageing and health [Internet]. Geneva: WHO; 2018 [capturado em 27 maio 2019]. Disponível em: http://www.who.int/mediacentre/ factsheets/fs404/en/.

28. Harridge SD, Lazarus NR. Physical activity, aging, and physiological function. Physsiology. 2017;32(2):152-61.

29. Grossman DC, Curry SJ, Owens DK, Barry MJ, Caughey AB, Davidson KW, Doubeni CA, Epling JW Jr, Kemper AR, Krist AH, Kubik M, Landefeld S, Mangione CM, Pignone M, Silverstein M, Simon MA, Tseng CW.
Interventions to prevent falls in community-dwelling older adults: US Preventive Services Task Force Recommendation Statement.. Jama. 2018;319(16): 1696-704. https://doi.org/10.1001/jama.2018.3097

30. Schlicht J, Camaione DN, Owen SV. Effect of Intense Strength Training on Standing Performance in Older Adults. J Gerontol. 2017;56(5):281-6.

31. Frontera WR, Bigard X. The benefits of strength training in the elderly. Sci Sport. 2002;17(3):109-16.

32. Lacroix A, Kressig RW, Muehlbauer T, Gschwind YJ, Pfenninger B, Bruegger O, Granacher U. Effects of a supervised versus an unsupervised combined balance and strength training program on balance and muscle power in healthy older adults: a randomized controlled trial. Gerontology. 2016;62(3):275-88. https://doi. org/10.1159/000442087

33. Vieira LS, Gomes AP, Bierhals IO, Farías-Antúnez S, Ribeiro CG, Miranda VIA, Lutz BH, Barbosa-Silva TG, Lima NP, Bertoldi AD, Tomasi E. Falls among older adults in the South of Brazil: prevalence and determinants. Rev Saúde Pública. 2018;52:1-13. https://doi.org/10.11606/ s1518-8787.2018052000103

34. Teixeira CS, Andrade RD, Barbosa DG, Alves RF, Felden EPG, Pedroso FS. Equilíbrio postural: investigação com crianças, adultos e idosos. Rev Bras Ciênc Envelhec Hum. 2015;12(2):134-46. https://doi.org/10.5335/rbceh. v12i2.5158

35. Maciel ÁCC, Guerra RO. Prevalence and associated factors to the balance alterations in elderly. Rev Bras Ciênc Mov. 2005;13(1):37-44.

36. Rodrigues EF, Souza DGR, Gazzola JM. Quedas no idoso com tontura. Rev Equilíbrio Corpor Saúde. 2011;3(2):40-4.

37. Macedo C, Gazzola JM, Ricci NA, Doná F, Ganança FF. Influence of sensory information on static balance in older patients with vestibular disorder. Braz J Otorhinolaryngol. 2015;81(1):50-7. https://doi.org/10.1016/j.bjorl.2014. 11.004

38. Terroso M, Rosa N, Marques AT, Simões R. Physical consequences of falls in the elderly: a literature review from 1995 to 2010. Eur Rev Aging Phys Act. 2013;11(1): 51-9. https://doi.org/10.1007/s11556-013-0134-8

39. Borges AP, Carneiro JA, Zaia JE, Carneiro AA, Takayanagui OM. Evaluation of postural balance in mild cognitive impairment through a three-dimensional electromagnetic system. Braz J Otorhinolaryngol. 2016;82 (4):433-41. https://doi.org/10.1016/j. bjorl.2015.08.023

40. Manna L, Kleinpaul JF, Teixeira CS, Rossi AG, Lopes LFD, Mota CB. Investigação do equilíbrio corporal em idosos. Rev Bras Geriatr Gerontol. 2008;11(2):155-65. https://doi.org/10.1590/1809-9823.2008.11023

41. Quitschal RM, Fukunaga JY, Ganança MM, Caovilha $\mathrm{HH}$. Evaluation of postural control in unilateral vestibular hypofunction. Braz J Otorhinolaryngol. 2014;80(4): 339-45. https://doi.org/10.1016/j.bjorl.2014.05.015 
42. Lemos LFC, David AC, Mota CB. Equilíbrio postural entre mulheres adultas em duas faixas etárias distintas. Rev Bras Ciência Mov. 2011;19(3):51-7.

43. Toledo DR, Barela JA. Diferenças sensoriais e motoras entre jovens e idosos: Contribuição somatossensorial no controle postural. Rev Bras Fisioter. 2010;14(3):267-75. https://doi.org/10.1590/s1413-35552010000300004

\section{AUTHORS:}

MARCELO de MAIO NASCIMENTO

Doutor em Ciências do Esporte. Professor da Universidade Federal do Vale do São Francisco (UNIVASF). Petrolina/Pernambuco, Brasil.

E-mail: marcelo.nascimento@univasf.edu.br

Orcid: https://orcid.org/0000-0002-3577-3439

Hugo daniel Gomez de CASTro

Discente do colegiado de Educação Física. Universidade Federal do Vale do São Francisco (UNIVASF). Petrolina/Pernambuco, Brasil.

E-mail: hugo.dgc@hotmail.com

Orcid: https://orcid.org/0000-0002-9873-2749

MATEUS Alves RAMOS

Discente do colegiado de Medicina. Universidade Federal do Vale do São Francisco (UNIVASF). Petrolina/Pernambuco, Brasil.

E-mail: mateusalvesramos2@hotmail.com

Orcid: https://orcid.org/0000-0002-0167-8660 